\title{
Gravitational and Electromagnetic Force Unification, Using a Statistical Analysis from a Signature of Supernova Explosions
}

\author{
Joseph Ngene Aniezi ${ }^{1, ~ *}$, Gabriel Anene ${ }^{1,2}$ \\ ${ }^{1}$ Department of Physics and Industrial Physics, Nnamdi Azikiwe University, Awka, Nigeria \\ ${ }^{2}$ Department of Physics, Legacy University, Okija, Nigeria
}

Email address:

aniezijoseph@gmail.com (J. N. Aniezi)

${ }^{*}$ Corresponding author

To cite this article:

Joseph Ngene Aniezi, Gabriel Anene. Gravitational and Electromagnetic Force Unification, Using a Statistical Analysis from a Signature of Supernova Explosions. International Journal of Astrophysics and Space Science. Vol. 7, No. 3, 2019, pp. 24-29.

doi: 10.11648/j.ijass.20190703.11

Received: July 17, 2019; Accepted: August 23, 2019; Published: September 6, 2019

\begin{abstract}
Unification of gravitational and electromagnetic interactions was done using statistical method. Cosmic ray intensity as a signature from supernova explosions was used to carry out some estimation. These primary cosmic rays are charged particles with an accelerating mechanism, probably electromagnetic; and because their charge is what interacts with matter and produces the effects that we can easily see here on earth. We estimated the values of the electromagnetic interaction and gravitational force of interaction of the source using previous known relations. We were able to obtain a regression equation; with a positive and strong correlation coefficient. $\mathrm{F}_{\mathrm{a}}$ depicts the gravitational interaction and $\mathrm{U}_{\text {maa }}$ is the electromagnetic interaction. The results suggestively indicate that gravitational and electromagnetic interactions are related. The result indicates that moving charges and moving masses provide an analogy of gravitational and electromagnetic fields, which support the concept of a gravito-magnetic force. It has solved to some extend the speculations about the development in an attempt to unify the interactions of particles; and even to incorporate gravity in the "theory of everything". Thus, the unification of forces using the explosion mechanism and gravitational waves in core-collapse supernovae has become a reality.
\end{abstract}

Keywords: Supernova Explosion, Cosmic Rays, Unification of Forces

\section{Introduction}

A unified theory of interaction, as it is generally understood implies a description of the four fundamental forces such as gravitation (between particles with mass), electromagnetic (between particles with charge/magnetism), strong interaction (between quarks) and weak interaction (operates between neutrinos and electrons). Based on the foundations of the Maxwell's equations, physicists are trying to combine the four fundamental forces in a single mathematical formulation. In the classical solutions of long ranged interactions, work is done in some other studies related to a unified equation [17].

The model at grand unified theories (GUT) implies that at high energy level of the universe, the four fundamental forces have the same validity. Therefore, the four fundamental forces can be written in a single representation to describe the true universe lepton [12].

There was a presentation on a theory of unification of gravitational force and the electromagnetic force based on generalization of Newton's law of gravitation to include a dynamic term inferred from the Lorentz force of electromagnetic interaction. The inclusion of this dynamic term alone in the gravitational force is enough to develop the entire dynamic theory of gravitation parallel to that of electrodynamics. The theory naturally solves the mystery of action-at-distance. It has been shown that the inverse square law of the static and the dynamic forces is the result of the conservation of mass (Gauss's Law) and the total momentum (Wang's Law). The Wang's Law is a new discovery. The new theory also predicts that the gravitational force is transmitted through propagation of gravitational waves at the speed of 
light [18].

The unification of gravity and electromagnetism is a conjecture of Einstein although he failed to show it because Einstein did not realize that this unification requires a new charge-mass interaction. Moreover, the existence of such an interaction has been verified by experiments. Apparently M. A. El-Lakany also fails to see the need of such a new interaction. Moreover, he also has no experiment predictions that can be used to support his theory. This has shown that Einstein's conjecture is valid [19].

The weak interactions are the results of the charged particles, and $\mathrm{W}$ and $\mathrm{Z}$ bosons are the force carriers of the weak interactions. The weak interactions are short ranged interactions [23]. Photons ( $)$ are the force carriers in the electromagnetic interactions, the electromagnetic interactions are the long ranged interactions [25]. The strong interaction is confined in the nucleus, it holds nucleons together, and three colored gluons $(\mathrm{g})$ are the force carriers in the strong interactions. The gravitational forces are the results of the mass effects of the particles and they are the long ranged interactions [5]. The non-relativistic Schrödinger equation is written in relativistic form by Dirac in 1928, so named as Dirac equation. It contains electromagnetic interactions of particles. Yukawa potential is used to describe the strong interactions at nuclei distances and the gravitational interactions at large distances [8].

There is quite remarkable disparity of strength among the different interactions. The strong interactions exceed the gravitational interactions in strength by the fantastically large factor of about $10^{41}$. The nuclear glue (strong interaction) makes the cosmic glue (gravity) look puny indeed. The electromagnetic interactions fall short of the strong interactions by a factor of about 100 , but exceed the weak interactions in strength by the enormous factor of $10^{11}$. The weak interactions in turn are about $10^{28}$ times stronger than the gravitational interactions. In fact, these numbers do not have a very precise meaning, but they indicate clearly that there exist vast gulfs between the strength of one interaction and another [26].

The electromagnetic force affects particles that have electric charge such as protons and electrons. This is the force that enables atoms to form by the electromagnetic attraction between the protons and the electrons which binds them together in atoms (like the gravity that makes the earth and other planets orbit around the sun). The forces have an infinite range. The particle that transmits the force is the virtual photons [12].

One of the four fundamental forces, the electromagnetic force manifests itself through the forces between charges (Coulomb's Law) and the magnetic force, both of which are summarized in the Lorentz force law. Fundamentally, both magnetic and electric forces are manifestations of an exchange force involving the exchange of photons. The quantum approach to the electromagnetic force is called quantum electrodynamics or QED. The electromagnetic force is a force of infinite range which obeys the inverse square law, and is of the same form as the gravity force [5]. The electromagnetic force holds atoms and molecules together. In fact, the forces of electric attraction and repulsion of electric charges are so dominant over the other three fundamental forces that they can be considered to be negligible as determiners of atomic and molecular structure. Even magnetic effects are usually apparent only at high resolutions and as small corrections [25].

The gravitational force is different from the other forces for three main reasons. The other three fundamental forces (electromagnetic, strong and weak interaction) affect only certain particles such as electron of quarks, but gravity affects all other particles. The gravitational force is very weak and can be considered as the weakest of all the forces. It makes this up by having, an electromagnetic force, at an infinite range. The nature of the force is attractive (meaning that it can only pull things towards it) [22]. Therefore, this has the implication that different gravitational sources can only add up to create a bigger source. This is different from the other forces which can be both attractive and repulsive, so they can also reinforce each other to create a bigger force, but they can also cancel each other out making a weaker force [28].

Weak interaction acts between quarks and leptons, whereas the strong force does not act between leptons because leptons are colourless and so do not participate in the strong interactions. Neutrinos have no charge, so they experience no electromagnetic forces but quarks, leptons and neutrinos join in the weak interactions [12]. The role of the weak forces in the transmutation of quarks, makes its interaction noticed in many decays of nuclear particles which require a change of a quark from one flavor to another. The weak interaction is the only process in which quark can change to another quark or a lepton to another lepton which is the so-called "flavor changes" [2].

Grand unification refers to unifying the strong interaction with the unified electroweak interaction. The basic problem of "restoring the broken symmetry" between the strong and electroweak forces is that the strong force works only on colored particles and the leptons don't have color. You have to be able to convert quarks to leptons and vice versa. But this violates the conservation of baryon number, which is a strong experimental nuclear physics principle [13]. The required mass of the exchange boson is $10^{15} \mathrm{eV}$, which is more like the mass of a visible dust particle than that of a nuclear entity. This particle is called the X-boson [5]. One prediction of the grand unified theories is that the proton is unstable at some level. The grand unification of the strong, weak, and electromagnetic forces is above $10^{14} \mathrm{GeV}$ of energy. If the ordinary concept of thermal energy is applied at such times, it would require a temperature of $10^{27} \mathrm{~K}$ for the average particle energy to be $10^{14} \mathrm{GeV}$ [25].

Recall that the electromagnetic and gravitational forces are normally very weak forces and the strong nuclear and weak nuclear forces are very strong forces. It was discovered that there was one level (as we go to smaller and smaller levels, the electromagnetic and gravitational force gets stronger while strong interaction and the weak interaction gets weaker). Since the strong and weak forces have gotten so weak, they can behave as the same force. But a lot of energy is required in the unification process of the fundamental 
forces before they can make a difference. Therefore, it requires very high temperatures before the forces start to act like one. Moreover, it seems like all forces were one and the same in the beginning of the universe. But then as everything cooled off, they branched of one by one to create the different forces we see today. But on second taught, it should be said that they become almost as the same force but not quite. For strong, weak, electromagnetic and gravitation to act exactly as the same force requires something called supersymmetry [14]. Supersymmetry means, that for every particle from one of the matter or force family, there is a corresponding particle from the other family but with half $(1 / 2)$ a spin different. The problem is that for supersymmetry to work, we ought to have a lot of particles, since every particle at the moment doesn't have a symmetry partner [1].

Although the above discussion indicates that the fundamental forces in our present universe are distinct and have very different characteristics, the current thinking in theoretical physics is that it was not always so. There is a rather strong belief (although it is yet to be confirmed experimentally) that in the very early universe when temperatures were very high compared with today, the weak, electromagnetic, and strong forces were unified into a single force. Only when the temperature dropped did these forces separate from each other, with the strong force separating first and then at a steady lower temperature did the electromagnetic and weak forces separate to leave us with the four distinct forces that we see in our present universe. The process of the forces separating from each other is called spontaneous symmetry breaking [11].

There is further speculation, which is even less firm than that above, that at even higher temperature (the Planck Scale), all the four forces were unified into a single force. Then, as the temperature dropped further, gravitation was the first to separate after which the other three forces separated as explained above. Theories that postulate the unification of the strong, weak, and electromagnetic forces are called Grand Unified Theories (often known by the acronym GUTs). Theories that add gravity to the mix and try to unify all four fundamental forces into a single force are called Superunified Theories. The theory that describes the unified electromagnetic and weak interactions is called the Standard Electroweak Theory, or sometimes just the Standard Model [13]. Grand Unified and Superunified Theories remain theoretical speculations that are yet unproven, but there is strong experimental evidence for the unification of the electromagnetic and weak interactions in the Standard Electroweak Theory. Furthermore, although GUTs are not proven experimentally, there is strong circumstantial evidence to suggest that a theory at least like a Grand Unified Theory is required to make sense of the Universe [31].

The discovery of the $\mathrm{W}$ and $\mathrm{Z}$ particles, the intermediate vector bosons, in 1983 brought experimental verification of particles whose prediction had already contributed to the Nobel Prize awarded to Weinberg, Salam, and Glashow in 1979. The photon, the particle involved in the electromagnetic interaction, along with the $\mathrm{W}$ and $\mathrm{Z}$ provide the necessary pieces to unify the weak and electromagnetic interactions [16]. With masses around 80 and $90 \mathrm{GeV}$, respectively, the $\mathrm{W}$ and $\mathrm{Z}$ were the most massive particles seen at the time of discovery while the photon is massless [21]. The difference in masses is attributed to spontaneous symmetry breaking as the hot universe cooled. The theory suggests that at very high temperatures where the equilibrium energies are in excess of $100 \mathrm{GeV}$, these particles are essentially identical and the weak and electromagnetic interactions were manifestations of a single force [3]. The question of how the $\mathrm{W}$ and $\mathrm{Z}$ got so much mass in the spontaneous symmetry breaking is still a perplexing one. The symmetry-breaking mechanism is called a Higgs field, and requires a new boson, the Higgs boson $(\mathrm{H})$ to mediate. The next step is the inclusion of the strong interaction in what is called grand unification [14].

The weak and electromagnetic fundamental forces seem very different in the present, relatively low temperature universe. But when the universe was much hotter so that the equilibrium thermal energy was on the order of $100 \mathrm{GeV}$, these forces may have appeared to be essentially identical, part of the same unified "electroweak" force [3]. The exchange particle for the electromagnetic part is the massless photon and the exchange particles for the weak interaction are the massive $\mathrm{W}$ and $\mathrm{Z}$ particles. The symmetry was spontaneously broken when the available energy dropped below $80 \mathrm{GeV}$ and the weak and electromagnetic forces taken on a distinctly different look. The model is that at an even higher temperature, there was symmetry or unification with the strong interaction which is the grand unification, and higher still, the gravity force may join to show the four fundamental forces to be a single unified force [10].

The concept of spontaneous symmetry breaking is important to the understanding of electroweak unification and further unifications [30]. In the snowflake, both the hydrogen and oxygen molecules are quite symmetric when they are isolated. The electric force which governs their actions as atoms is also a symmetrically acting force. But when their temperature is lowered and they form a water molecule, the symmetry of the individual atoms is broken as they form a molecule with 105 degrees between the hydrogen-oxygen bonds. When they freeze to form a snowflake, they form another type of symmetry, but the symmetry of the original atoms has been lost. Since this loss of symmetry occurs without any external intervention, we say that it has undergone spontaneous symmetry breaking [20]. A magnet can be used as an analogy to illustrate the concept of spontaneous symmetry breaking which is important to the understanding of electroweak unification and further unifications. When the magnet is strongly magnetized in one direction, it would be hard to guess that the underlying interaction is actually symmetric under rotation. The magnetic field from the magnet is certainly very different if it is rotated 90 degrees, or 180 degrees [4]. The underlying symmetry can only be seen if the energy of the system is raised; heating the magnet to its Curie temperature would remove the directional magnetic field and restore the 
rotational symmetry of the material. This is an apt analogy for the electroweak unification, since the symmetry between the Coulomb force and the weak interaction is certainly not evident at low temperatures [29]. However, above unification energy, on the order of $100 \mathrm{GeV}$, they would merge into a single electroweak force [7]. Electroweak theory is very important for modern cosmology, particularly on how the universe evolved. This is because shortly after the big-bang, the temperature was approximately above $10^{15} \mathrm{~K}$ because electromagnetic force and weak force were merged into a combined electroweak force [12].

Supernova explosions of massive stars are an important phenomenon for applying particle physics, in particular astrophysics. The shockwaves of supernova explosions accelerate charged particles such as protons; some of which end up raining on Earth as cosmic rays [24]. Primary Cosmic Rays are stable charged particles that have been accelerated to enormous energies by astrophysical sources somewhere in our universe. They must be stable (lifetimes greater than a million years), in order to survive the long trips through interstellar (or intergalactic) space. They are charged because the accelerating mechanism is probably electromagnetic and because their charge is what interacts with matter and produces the effects that we can easily see here on earth. They have a range of energies, $10^{9} \mathrm{eV}(1 \mathrm{GeV})$ up to $10^{20} \mathrm{eV}$ $\left(10^{8} \mathrm{TeV}\right)$. Cosmic ray particles perform wandering in the tangled magnetic fields of the Galaxy [6]. The expanding stellar debris creates a shockwave when it slams into the surrounding gas, compressing and enhancing any magnetic fields present. A major factor affecting the path of cosmic rays is that they are charged and therefore swerved by magnetic fields. This most definitely affects any attempts to locate sources. The more energy a particle has, the greater the radius of curvature. Particles with energies less than $10^{15} \mathrm{eV}$ have paths that are so curved that they are probably trapped within our galaxy and wander throughout [15]. In this work, we shall unify gravitational and electromagnetic forces using a statistical approach from a signature of supernova explosions.

\section{Materials and Method}

Mexico City Observatory (http://132.248.105.25/index hp) is the source of cosmic ray intensity data, emanating from supernova explosions. The cosmic ray intensity was collected for a period of twelve months, for the year 2017. It was used to carry out some estimation; and tried using statistical data analyses to obtain a relation that suggestively unify gravitational and electromagnetic interactions.

From the work of Robson, 1996, we obtained the magnetic field energy density $U_{\text {mag }}$ using:

$$
U_{m a g}=\frac{3}{4 \sigma_{T} c \gamma^{2}} \cdot\left(\frac{E}{t}\right)=\left(3.759 \times 10^{19}\right) \cdot\left(\frac{E}{t}\right)
$$

where $E$ is energy in Joule:

$$
\left(E=\frac{4}{3} \sigma_{T} c \gamma^{2} U_{m a g} \cdot t=\left(2.66 \times 10^{-20}\right) . U_{m a g} \cdot t\right)
$$

$t$ is time in second. The time in days for each of the twelve (12) months were converted to second:

$$
t_{\text {second }}=\left(t_{\text {days }} \times 24 \times 60 \times 60\right)
$$

$\sigma_{T}$ is Thompson cross-section, $c=3.0 \times 10^{8} \mathrm{~m} / \mathrm{s}$ is the speed of light, $\gamma=1$ is Lorentz factor at $v \ll c$. We assumed bolometric luminosity of the source, and $\sigma_{T}=6.65 \times 10^{-29} \mathrm{~m}^{2}$. Equation (1) simply indicates that we can estimate the value of the source magnetic field energy density once it is bolometric luminosity and jet speed are known. The estimates are shown in Table 1.

The mass of blackhole was estimated from Albert Einstein's equation:

$$
m_{b}=\frac{E}{c^{2}}
$$

Moreover, gravitational force $F_{g}$ of interaction of a given blackhole is given by:

$$
F_{g}=2 \times 10^{-6} \frac{\pi k}{\hbar} \frac{m_{\odot}}{m_{b}}=\frac{\left(1.6377 \times 10^{36}\right)}{m_{b}}(\text { Hawking, 1976) }
$$

Where

$$
k=1.381 \times 10^{-23} \mathrm{~m}^{2} \mathrm{kgs}^{-2} \mathrm{~K}^{-1}
$$

is Boltzmann constant, $m_{\odot}=1.989 \times 10^{30} \mathrm{~kg}$ is solar mass, $m_{b}$ is mass of blackhole. Using equation (3), we estimated

\begin{tabular}{|c|c|c|c|c|c|c|c|c|}
\hline MONTH & CR LUMINOSITY $\left(\frac{d E}{d t}\right)$ & TIME (Seconds) & $\begin{array}{l}U_{m a g} \\
X 10^{27} \\
\end{array}$ & $\begin{array}{l}E(J) \\
X 10^{-13}\end{array}$ & $\begin{array}{l}m_{b}(\mathrm{~kg}) \\
X 10^{-30}\end{array}$ & $\begin{array}{l}F_{g}(\mathrm{~N}) \\
X 10^{65}\end{array}$ & $\log U_{m a g}$ & $\log F_{g}$ \\
\hline Jan. & 55779580 & 2678400 & 2.0970 & 1.4940 & 1.6600 & 9.8657 & 27.3216 & 65.9941 \\
\hline Feb. & 42020423 & 2419200 & 1.5795 & 1.0164 & 1.1293 & 14.5019 & 27.1985 & 66.1614 \\
\hline March & 58155787 & 2678400 & 2.1861 & 1.5575 & 1.7306 & 9.4632 & 27.3397 & 65.9760 \\
\hline April & 53860424 & 2592000 & 2.0246 & 1.3959 & 1.5510 & 10.5590 & 27.3063 & 66.0236 \\
\hline May & 53892206 & 2678400 & 2.0258 & 1.4433 & 1.6037 & 10.2120 & 27.3066 & 66.0091 \\
\hline June & 45941213 & 2592000 & 1.7269 & 1.1906 & 1.3229 & 12.3796 & 27.2373 & 66.0927 \\
\hline July & 61550921 & 2678400 & 2.3137 & 1.6484 & 1.8316 & 8.9414 & 27.3643 & 65.9514 \\
\hline August & 45620575 & 2678400 & 1.7149 & 1.2218 & 1.3576 & 12.0632 & 27.2342 & 66.0815 \\
\hline
\end{tabular}
gravitational force of interaction of the source and the values are shown in Table 1 . Scatter plot of logarithmic scale of $F_{g}$ and $U_{m a g}$ was carried out and is shown in figure 1.

\section{Results}

Table 1. Estimated Data from 2017 Cosmic Ray (CR) Intensity. 


\begin{tabular}{|c|c|c|c|c|c|c|c|c|}
\hline MONTH & CR LUMINOSITY $\left(\frac{d E}{d t}\right)$ & TIME (Seconds) & $\begin{array}{l}U_{m a g} \\
X 10^{27} \\
\end{array}$ & $\begin{array}{l}E(J) \\
X 10^{-13} \\
\end{array}$ & $\begin{array}{l}m_{b}(\mathbf{k g}) \\
X 10^{-30} \\
\end{array}$ & $\begin{array}{l}F_{g}(\mathrm{~N}) \\
X 10^{65}\end{array}$ & $\log U_{m a g}$ & $\log F_{g}$ \\
\hline Sept. & 59004294 & 2592000 & 2.2180 & 1.5292 & 1.6991 & 9.6386 & 27.3460 & 65.9840 \\
\hline Oct. & 55313353 & 2678400 & 2.0792 & 1.4813 & 1.6459 & 9.9502 & 27.3179 & 65.9978 \\
\hline Nov. & 49634110 & 2592000 & 1.8657 & 1.2863 & 1.4292 & 11.4589 & 27.2708 & 66.0591 \\
\hline Dec. & 53699615 & 2678400 & 2.0186 & 1.4382 & 1.5980 & 10.2484 & 27.3051 & 66.0107 \\
\hline
\end{tabular}

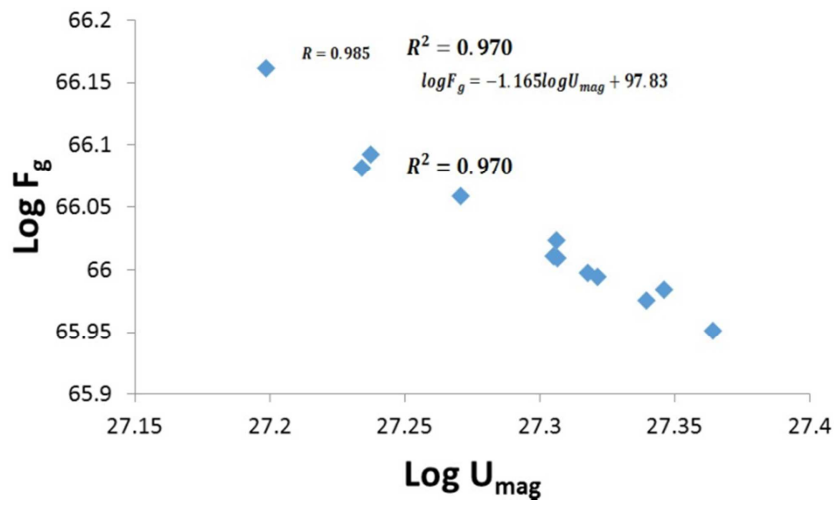

Figure 1. Plot of Gravitational and Electromagnetic Interaction and Unification.

From the plot of gravitational and electromagnetic interactions, the equation of regression gives:

$$
\log F_{g}=-1.165 \log U_{m a g}+97.83
$$

with a good correlation coefficient $(R=0.985)$.

Simplifying equation (4) further, we took anti-log on both sides, to obtain:

$$
F_{g}=10^{\left(-1.165 \log U_{m a g}+97.83\right)}
$$

Opening the bracket of the expression, we obtained:

$$
F_{g}=10^{\left(-1.165 \log U_{m a g}\right)} \times 10^{97.83}
$$

Hence, equation (6) becomes:

$$
F_{g}=\left(6.76 \times 10^{97}\right) \cdot 10^{-1.165 \log U_{m a g}}
$$

Rearranging equation (7), we obtained:

$$
F_{g}=\left(6.76 \times 10^{97}\right) \cdot 10^{\log U_{m a g}^{-1.165}}
$$

Therefore, the relation becomes:

$$
F_{g}=\left(6.76 \times 10^{97}\right) \cdot U_{m a g}^{-1.165}
$$

Equation (9) simply suggested that $F_{g}$ varies with $U_{\text {mag }}$ according to the relation:

$$
F_{g} \sim U_{m a g}^{-\Psi}
$$

where $\Psi=1.165$ is the slope of the plot.

\section{Discussion}

The unification was conducted using statistical method. Statistical data was obtained from the Mexico City Observatory, where we estimated the values as shown in table 1. A plot of $F_{g}$ against $U_{m a g}$ gives a regression equation: $\log F_{g}=-1.165 \log U_{m a g}+97.83$; and with a good correlation coefficient, $R=0.985$. There is a positive and strong relationship between the gravitational interaction $F_{g}$ and the electromagnetic interaction, $U_{m a g}$. This suggestively indicate that gravitational and electromagnetic interactions relate according to the expression: $F_{g} \sim U_{\text {mag }}^{-\psi}$; where $\Psi=1.165$ is the slope of the plot. The electromagnetic force, $U_{m a g}$ affects the cosmic ray particles that have electric charge such as electrons. This is the force that enables the atoms to form by the electromagnetic attraction between the protons and the electrons which binds them together in atoms.

The result in equation (10) indicates that moving charges and moving masses provide an analogy of gravitational and electromagnetic fields, which support the concept of a gravito-magnetic force. This has attempted to solve to some extend the speculations about the development in an attempt to unify the interactions of particles; and even to incorporate gravity in the "theory of everything". Thus, the unification of forces using the explosion mechanism and gravitational waves in core-collapse supernovae is now becoming reality.

In comparison, Wagener (2009) limited its scope on unifying the three fundamental forces: Gravitation, Electrodynamics and the strong force with the help of the derivation of the Yukawa potential. Ling, 2018 presented a theory of unification of gravitational force and the electromagnetic force based on generalization of Newton's law of gravitation to include a dynamic term inferred from the Lorentz force of electromagnetic interaction.

In this work, forces were unified with the use of statistical analysis of cosmic ray intensity as a signature of supernova explosions. By "unify" we mean finding connections between the gravitational and electromagnetic forces, which show that they are different manifestations of a single force.

\section{Conclusion}

The unification process obtained for gravitational and electromagnetic interaction, accounted for a good correlation coefficient which indicates validity of the both interaction. It connects the electromagnetic field with gravity. The unification of forces using the explosion mechanism and gravitational waves in core-collapse supernovae has become a reality. It also provides insight into the unification of the fundamental interactions, which in addition provides detailed information about the universe. Future researchers should use other extragalactic sources/data in their investigations. More researches should be carried out using the statistical data analysis rather than analytical approach to enhance the research on unification of fundamental forces/super- 
unification.

\section{Acknowledgements}

A profound gratitude goes straight to God for His sustenance. Thanks go to Prof. G. Anene for contributing immensely in this research work. We would also like to acknowledge the immense support of the Mexico City Observatory for providing the data used for this research. We remain greatly indebted.

\section{References}

[1] Assis, A. K. T. (2002). On the Unification of forces of Nature. Annales de la Fondation Louis de Broglie, 27 (2): 149-160.

[2] Borzor, I. N. and Goriely, S. (2000). Weak Interaction Rates of Neutron-Rich Nuclei and the R-Process Nucleosynthesis. Physical Review, 62: 1-12.

[3] Collins, P. O. B., Martin, A. D. and Squires, E. J. (1989) Particle Physics. John Wiley and Sons, England. 1-171.

[4] Gamba, A., Marshak, R. E. and Okubo, S. (1959). A Symmetry in Weak Interactions Physics Physical Review, 45: 881-885.

[5] Giancoli, C. D. (2000). Physics of Scientist and Engineers with Modern Physics. Prentice Hall, Upper Saddle River Publisher, New Jersey. 586- 1134.

[6] Ginzburg, V. and Syrovatskii, L. (1969). The Origin of Cosmic Rays. New York: Gordon and. Breach.

[7] Glashow, S. L. (1960). Partial-Symmetries of Weak Interaction; Nuclear Physics 22: 579-588.

[8] Hasan, A. (2011). A Unified Equation of Interactions. Open Journal of Microphysics, 1 (28): 28-31.

[9] Hawking, S. W. (1976). Blackholes and Thermodynamics. Physical Review D 13: 2-7.

[10] Howard, G. and Glashow, S. L. (1974). Unity of all Elementary-Particle Forces. Physical. Review Letter, 32 (8): 440-441.

[11] Hroost, P. W (1966). Spontaneous Symmetry Breakdown without Massless Bosons. Physical. Review, 145: 4-9.

[12] Hugh, D. Y. and Roger, A. F. (2004). University Physics. Dorling Kindersley Ltd. Publisher, India. 1469-1475.

[13] Hughes, I. S. (1985). Elementary Particles (Second Edition). Press Syndicate of the University of Cambridge Publisher USA. 55-302.

[14] Isaev, P. S. (1989). Quantum Electrodynamics at High Energy. American Institute of Physics Inc Publisher, USA. 1-46.
[15] Kang, H., Ryu, D. and Jones, T. W. (1996). Cluster Accretion Shocks as Possible Acceleration Sites for Ultra High Energy Protons below the Greisen Cuto. ApJ, 456, 422.

[16] Ken-ichl, A., Zenro, H., Rokuo, K., Michij, I. and Taizo, M. (1982). Electroweak. Theory Supplement of the Progress of Theoretical Physics, 73: 4-10.

[17] Lalit, K. S., Pearson, V. L. and Samuel, C. (2011). Potentials for the Klein-Gordon and Dirac. Equations. Chiang Mai J. Sci. 38 (4): 514-526.

[18] Ling, J. W. (2018). Unification of Gravitational and Electromagnetic Forces. Fundamental Journal of. Modern Physics. ISSN: 2249-9768. Vol. 11, Issue 1, 2018, Pages 29-40. http://www.frdint.com/

[19] Lo, C. Y. (2017). Comments on "Unification of Gravity and Electromagnetism by Mohammed A.. El-Lakany" \& Einstein's Unification. Journal of Physical Science and Application. 7 (4) (2017). 28-32. doi: 10.17265/21595348/2017.04.003.

[20] Lowell, S. B. (1992). Quantum Field Theory. Press Syndicate of the University of Cambridge, USA. 98-105.

[21] Mike, G. (1991). Gauge Field Theories. John Wiley and Son Inc. Publisher, England. 266-328.

[22] Minkevich, A. V. (2009). Gravitational Interaction and Poin Care Gauge Theory of Gravity. Acta Physica Polonica B, 40 (2): $1-10$

[23] Orear, J. (1979). Physics. Macmillan Co. Inc., Publisher, England. 292-710.

[24] Rao, M. (1998). Extensive Air Showers, World Scientific, p. 10, ISBN 9789810228880.

[25] Renton, P. (1990). Electroweak Interaction: An Introduction of the Physics of Quarks and Lepton University of Cambridge Publisher, USA. 1-544.

[26] Resnick, W. B. (1993). The Fundamental Particles and their Interaction. Lachina Services Publisher, USA. 163-371.

[27] Robson, I. (1996). The Physics and Evolution of Active Galactic Nuclei. Physical Review, 75 (2): 118-122.

[28] Ryuichiro, K. (2006). Gravitational Gauge Mediation. Physics Letters B, 641: 203-207.

[29] Salam, A. (1980). Gauge Unification of Fundamental Forces. Reviews of Modern Physics, 52 (3): 525- 536.

[30] Steven, W. (2000). The Quantum Theory of Fields. The Press Syndicate of the University of Cambridge, USA. 198-235.

[31] Sundaresan, M. K. (2001). Handbook of Particle Physics. Library of Congress Cataloging-In-Publication Data, USA. 131-362.

[32] Wagener, P. (2009). A Unified Theory of Interaction: Gravitation, Electrodynamics and the. Strong.. Force. Progress in Physics 1: 33-35. 